\title{
GENOME ORGANIZATION AT DIFFERENT SCALES: NATURE, FORMATION AND FUNCTION
}

Jacques Serizay and Julie Ahringer

The Gurdon Institute and Department of Genetics, University of Cambridge, Cambridge CB2 1QN, United Kingdom

Author for correspondence: ja219@cam.ac.uk 


\begin{abstract}
Since the discovery of chromosome territories, it has been clear that DNA within the nucleus is spatially organized. During the last decade, a tremendous body of work has described architectural features of chromatin at different spatial scales, such as A/B compartments, Topologically Associated Domains (TADs), and chromatin loops. These features correlate with domains of chromatin marking and gene expression, supporting their relevance for gene regulation. Recent work has highlighted the dynamic nature of spatial folding and investigated mechanisms of their formation. Here we discuss current understanding and highlight key open questions in chromosome organization in animals.
\end{abstract}




\section{Introduction}

The current view of nuclear organization has come predominantly from applying variations of two major types of method, (i) microscopic observations or (ii) assessment of chromatin interactions using Chromosome Conformation Capture (3C) techniques (see [1] for a concise review of these methods). Using microscopy to visualise fluorescent probes targeted to specific loci can reveal the spatial location of whole chromosomes and the relative positions of loci with respect to each other or to landmarks such as the nuclear envelope or nucleoli. On the other hand, 3C methods detect interactions between two regions of chromatin [2]. In these methods, the physical proximity of two regions of DNA within the nucleus is inferred from the frequency of ligation events generated between them following nuclear fixation and digestion. Although captured ligation events are referred to as "chromatin interactions," in reality they represent regions of DNA that were close enough to be ligated together, which could be because of a direct interaction between these regions or because the regions occupied the same general vicinity. Applying the 3C technique genome-wide (Hi-C), chromatin interactions can be mapped across the genome [3], with resolution related to the depth of sequencing $[3,4]$. Importantly, $3 \mathrm{C}$ methods and microscopy are highly complementary. 3C methods identify putative chromatin interactions usually from cell populations and cannot assess the frequency of occurrence of the identified interactions across the population. On the other hand, microscopy can be used to validate interactions and their frequency, by visualising large numbers of individual nuclei. Live imaging is also powerful to investigate the stability of interactions and the dynamics of the association of proteins with chromatin.

Applying these methods has led to the definition of different types of chromatin organization, such as chromosome territories, compartments, TADs, insulated domains, contact domains, and loops. Here we discuss their properties and potential relationships. 


\section{Large-scale organization: chromosome territories and}

\section{compartments}

The initial visualization of the spatial positioning of chromosomes by microscopy demonstrated that their organization is actively regulated within the nucleus. Individual chromosomes are spatially organized in interphase nuclei, occupying distinct chromosome territories (CTs), and adopting relatively reproducible positions in different cells with limited intermingling (Figure 1A). Additionally, inactive regions of chromatin are often found in proximity to the nuclear envelope whereas active chromatin generally has a more internal position within the nucleus [5].

More recently, 3C-based procedures have been instrumental in assessing 3D structure of individual chromosomes at increasingly higher resolution. Using Hi-C to derive average chromosome conformations from capturing pair-wise interactions in populations of cells revealed that chromosomes have two major types of structural domains, termed A and B compartments [3]. The A compartment contains active chromatin (denoted by transcriptional activity, higher chromatin accessibility and H3K36me3 deposition) while the B compartment, more compacted, is associated with inactive chromatin (denoted by low transcriptional activity, association with the nuclear lamina and H3K27me3 deposition) [3,4]. Importantly, the plaid pattern obtained by plotting pair-wise correlation scores of interaction landscapes, when observed across entire chromosomes (Figure 1B), reveals that chromatin interactions are more frequent between regions of the same compartment type (A with A, and B with B) [3]. A recent Hi-C study conducted on single mammalian cells provided striking views of the spatial arrangements of A and B compartments [6]. In modelling the arrangement of all chromosomes within the nucleus, it was shown that DNA from the A compartment is organized in an inner ring-shaped structure, while DNA from the B 
compartment preferentially associates with the lamina and the edges of nucleoli (Figure 1A). These results are consistent with previous studies that used microscopy to map the locations of active and inactive chromatin within nuclei $[5,7]$.

A single-cell Hi-C study also highlighted the stochastic positions of A and B compartments in interphase cells [6]. Although a locus on a given chromosome occupies the same compartment in different nuclei, the spatial folding of the chromosome varies between nuclei (Figure 1A). This is in agreement with the finding that positions of lamina-associated chromatin (largely corresponding to the B compartment) are not heritable. Instead, these regions are randomly redirected to the nuclear lamina or near nucleoli after mitosis, with some of them switching from a nuclear lamina position to a nucleolar associated location [8]. These studies show that chromosomes have different conformations in different cells and that A compartment active chromatin and B compartment inactive chromatin are spatially segregated both within chromosomes and globally within nuclei.

Importantly, A/B compartment organization is only observed in interphase. During mitosis, chromatin structure is radically rearranged (Figure 1C) $[5,9,10]$. Hi-C studies performed on synchronised cells showed that minutes after entering prophase, chromosomes lose $\mathrm{A} / \mathrm{B}$ compartment organization and progressively generate and compact arrays of loops arranged around helical scaffolds of condensin I and II complexes (REF). This raises the question of how compartment structure is reformed.

Although a relationship between transcriptional activity and compartments is clear, the mechanism of compartment formation and function are not yet understood. A striking feature of $\mathrm{A}$ and $\mathrm{B}$ compartments is their different chromatin composition, including histone modifications associated with gene activity or inactivity, respectively. Chromatin state domains, which are defined by differently marked chromatin, have been noted to subdivide the genomes of animals, and their position in the genome is relatively constant during 
development [11]. Interestingly, super-resolution imaging has shown that different chromatin state domains (e.g., active, inactive, Polycomb marked) have distinct types of 3D organization, with Polycomb-marked chromatin having the densest packing $[12,13]$. Furthermore, altering local chromatin composition through targeting histone modifiers can drive repositioning to different compartments [14]. Whereas histone modifications can be inherited through cell division, most compartment interactions are lost during mitosis but regained after division $[9,15]$ (Figure 1C). These data suggest a model where the formation and structure of chromosome compartments relies on chromatin domains [16-18]. In such a model, chromatin reorganization that occurs during mitosis would prevent $\mathrm{A} / \mathrm{B}$ compartment interactions, while retention of chromatin domain marking would provide a framework for regenerating compartments in daughter cells (Figure 1C).

What might cause the segregation of chromatin into two types of spatial compartment? A growing body of work has shown that liquid-liquid phase separation can drive the formation of non-membrane bound compartments in the nucleus and cytoplasm [19]. For instance, the nucleolus is a phase separated compartment containing several different immiscible liquid-like sub-compartments, and HP1 containing heterochromatin has liquid-like properties and appears to form by phase separation [20-23]. The formation of these membrane-less compartments is thought to be driven by the local condensation of proteins containing unstructured regions. It is plausible that domains of particular chromatin modifications and/or proteins could drive phase-separated compartments that organize chromosome structure.

\section{Intermediate scale organization: Topologically Associated}

\section{Domains}

At a more local scale, chromatin interaction studies mostly in Drosophila and mammalian 
cells have described the segmentation of the genome into small physical domains of tens of kilobases up to a few megabases, and generally containing a small number (e.g., 1-10) of genes [4,24-28]. These self-interacting domains are variously termed "Topologically Associated Domains" (TADs) [24-26], sub-TADs [27], "contact domains" [4] and "insulated neighbourhoods" [28]. They are defined based on observing frequent chromatin interactions within a region and relatively fewer interactions with neighbouring chromatin. Because these differently named domains are defined in a similar way, and it is unknown whether they are functionally different, we will refer to this class of chromosome segmentation domain as "topologically associated domains" (TADs) without distinction. The properties of TADs support the view that they represent functional domains. For example, histone modification and replication timing are often similar across individual TADs [4,29]. Additionally, TADs appear to constrain the regulatory activity of enhancers [30].

\section{TAD boundaries}

The positions of TAD boundaries defined from studies on populations of cells appear relatively conserved in different cell types and across evolution [27,28,31-34]. In mammals, TAD boundaries interact more frequently with each other than with any other locus within the TAD and usually show binding of the CCCTC binding factor CTCF and the cohesin complex $[4,24,25]$. CTCF was initially identified as a protein with insulator activity, and its binding motifs at interacting boundaries are almost always oppositely oriented $[4,34,35]$. These observations have led to the notion that a chromosome domain is constrained within an insulating loop anchored by oppositely oriented CTCF proteins at the two boundaries of the domain (Figure 2). This model is supported by the analyses of mutants with deletions or inversions of CTCF sites at TAD boundaries, which led to predicted fusions or alterations of TADs [36,37]. 
The importance of TAD domain organization is also supported by gene expression and phenotypic alterations that are associated with TAD perturbations. In late embryonic development in the mouse, deleting a boundary between TADs that separate Hox genes alters gene expression and leads to skeletal defects [38]. In human and mouse, the inversion, deletion or duplication of TADs or TAD boundaries was shown to alter expression of genes located in the affected TADs, resulting in heart or limb pathologies $[39,40]$, Cook syndrome [41] or cancer susceptibility [42].

\section{Mechanism and dynamics of domain formation}

The cohesin complex forms a ring structure that entraps DNA for sister chromatid cohesion in meiosis and mitosis [43]. The enrichment of cohesin at TAD boundaries in interphase cells, together with its ability to entrap DNA, has led to a "loop extrusion" model to describe the formation of insulating loops $[44,45]$ (Figure 3 ). In this model, a loop of DNA is dynamically extruded by a loop extrusion factor (LEF) that contains cohesin (Figure 3b-f). Encountering a "boundary factor" (BF) such as CTCF would stabilize the complex (Figure 3e

- i). This model would explain the enrichment of cohesin and CTCF at TAD boundaries and the strong interaction signal observed between these regions. Of note, consistent with these roles, cohesin binding is located on the inner edge of the TAD relative to CTCF (Figure 3e) [46].

Increasing experimental and modelling studies have given strong support to the involvement of cohesin and loop extrusion in regulating chromosome organization (see [47] for a recent review). However, their mechanisms are still unclear. For example, the factors or processes providing the force for loop extrusion are not yet known. Transcriptional activity is correlated with TADs, and a recent computational model suggests that the negative supercoiling generated by transcription could provide energy for loop extrusion by 'pushing' 
cohesin handcuffs $[22,45,48]$. However, TADs may not rely on transcription, as they start forming in Drosophila embryogenesis before the onset of the majority of zygotic transcription, and still form even after chemical inhibition of RNA polymerase [49,50].

The dynamics of cohesin and CTCF binding to chromatin argue that loops are not static structures but instead are constantly forming and collapsing (Figure $3 \mathrm{~d}-\mathrm{f}$ ). Cohesin has a residence time of $\sim 22$ minutes, and CTCF, potentially playing the role of an insulating loop anchor, has a residence time of $\sim 1$ minute [51]. This implies that cohesin/CTCF loops are present only transiently even when ends are at TAD boundaries (Figure 3). The binding dynamics also explains how an extruding loop could bypass a TAD boundary to form a larger loop. Finally, dynamic binding suggests that nested extrusion would be expected to form within existing loops. A dynamic nature of chromosome domains is also supported by singlecell Hi-C studies $[6,52,53]$. Although averaged TAD boundary positions converge to those defined using a large number of cells, individual cells differ in TAD positions, and TADs can transgress conserved TAD boundaries. These studies support the view of dynamic loop formation and collapse and indicate that TADs are not stable structures (Figure 3).

\section{Factors involved in the formation of domains and boundaries}

A series of recent studies directly investigated the roles of cohesin and CTCF in interphase chromosome organization by removing them in mammalian cells [16,54]. Loss of CTCF, the Rad21 component of cohesin, or the cohesin loading factor Nipbl, led to the loss of TADs and loops $[16,54,55]$, underlining the important structural role of both CTCF and cohesin in forming loops and insulated domains. In line with these results, the cohesin release factor WAPL was shown to restrict loop extension, as evidenced by the increase in loop size upon its depletion [56]. However, although loops and TAD structure were lost upon CTCF or cohesin removal, A/B compartment structure remained intact, indicating that TADs and 
compartments are two independent types of structure $[16,54,55]$. CTCF or cohesin loss did not cause widespread transcriptional changes but only affected the expression of a limited set of genes, suggesting that much of normal gene expression is not dependent on TAD structure. It may be that compartments, which are retained, are important in this context.

The regulation of nucleosome dynamics at TAD boundaries also has the potential to control boundary "strength" (i.e. the level of segregation of interactions on each side of the boundary). TAD boundaries are sensitive to DNAse I digestion which indicates a lower nucleosome density [57,58]. Moreover, loss of the nucleosome remodelling protein BRG1 increases nucleosome occupancy at TAD boundaries and reduces boundary strength and CTCF binding [59]. In addition to affecting the binding of boundary factors, nucleosome dynamics has the potential to affect boundary function through changing local chromatin flexibility (see [60] for further discussion).

Importantly, factors involved in domain formation appear to differ in different animals. Mammals show strong CTCF/cohesin loop anchors at TAD boundaries [4,27] whereas in Drosophila, CTCF sites are at a small proportion of TAD boundaries and are not usually in inverted orientation [18]. Instead, Drosophila TAD boundaries are enriched for a number of other architectural proteins, such as CP190 and BEAF [57,58]. Furthermore, recent studies indicate that the prevalent strong loop anchors observed in mammals do not exist in Drosophila and that many TAD "boundaries" are instead domains of active genes $[18,57,61]$.

\section{Domains in other organisms}

The widespread TAD structure described in mammals and Drosophila has not been observed in other organisms such as C. elegans [62] and A. thaliana [63]. However, this difference may be due to technical and/or biological limitations, such as Hi-C map resolution and gene 
spacing. Notably, TAD-like structures are visible in gene-depleted regions of these otherwise compact genomes [18]. Although TADs are not apparent in C. elegans, a larger domain structure required for dosage compensation has been observed on the $\mathrm{X}$ chromosome [62]. Additionally, C. elegans autosomes are demarcated by alternating chromatin domains of H3K27me3 and H3K36me3 which contain genes with different modes of regulation $[11,15]$. Although the relationship between this chromatin domain pattern and spatial organization is not yet known, a similar chromatin domain organization of high versus low levels of H3K27me3 occurs in Drosophila sperm, and this pattern aligns well with TADs and TAD boundaries, respectively [17,61]. The alignment of histone modification domains with TADs together with the finding that compartments and histone modification patterns are not generally affected by loss of cohesin or CTCF in mammals suggests that chromatin domains may provide a primary level of 1D chromatin organization and regulation upon which higherlevel organizational mechanisms act.

\section{Small-scale chromatin interactions}

Variant 3C methods such as 4C, 5C, ChIA-PET or promoter capture, focusing on selected regions of the genomes, have uncovered extensive contacts between regulatory elements (i.e. promoters and enhancers), especially within TADs, which are not generally visible using genome wide methods such as Hi-C [64-67]. Enhancers usually contact multiple promoters and vice versa (Figure 2), and interacting regions show correlated activity, suggesting that contacts have functions in transcriptional control. Some genomic regions, such as Frequently Interacting REgions (FIREs) show particularly dense local interactions [31,68] and are associated with networks of co-expressed tissue-specific genes clustered within the same domain [68]. Their function is not yet known, but they might serve as a platform for transcription regulation in a domain. The anchors of enhancer/promoter interactions are less 
enriched for the combination of CTCF and cohesin compared to loop anchors at insulating TAD boundaries suggesting alternative mechanisms for their formation $[4,27,31,40]$. This observation could explain the relatively weak effect of CTCF and cohesin depletion on gene regulation $[16,54]$.

There is evidence that both pre-established loops and de novo loop formation play roles in regulating transcriptional output. In Drosophila and mammals, interactions between enhancers and promoters are detected before gene activation and are associated with paused RNA polymerase, suggesting that such contacts prime later expression $[31,64,69]$. Similarly, during early neural lineage commitment, enrichment of transcription factor YY1 at a set of pre-established regulatory loops is associated with transcription activation [33]. During macrophage development, transcription activation is associated with both the formation of new regulatory loops and increased acetylation of H3K27 at pre-existing loop anchors [31]. Finally, directly inducing contact between an enhancer and a promoter can drive transcription, supporting the functionality of interactions [70,71].

In summary, the current data support roles for chromatin interactions in regulating gene expression and controlling chromosome organization. Yet the mechanisms that govern patterns of regulatory element interactions are still poorly understood.

\section{Conclusion}

In this review, we have highlighted the diverse and versatile mechanisms implemented within the nucleus to build spatially organized and regulated chromatin. Although recent work has provided a remarkable improvement in our understanding of genome organization, many outstanding questions remain, such as 1) How are higher-order structures such as A/B compartments formed? Do liquid-liquid phase transitions play a role? 2) How are TADs formed? What provides the force for loop extrusion? 3) How are contacts between regulatory 
elements made and what are their functions? What are the roles of transcription factors? 4) How many different types of loop exist, and what are their functions?

The increasing use of perturbation analyses, studies of protein and regulatory dynamics, and investigations at higher resolution will help to address these and other fundamental questions. The field is at an exciting stage where new studies and technologies should lead to breakthroughs in our understanding of genome regulation and organization.

\section{Acknowledgments}

We thank Wei Qiang Seow, Tessa Gaarenstroom and Andrea Frapporti for their thoughtful comments on the manuscript. JS was supported by a Medical Research Council DTP studentship and JA by a Wellcome Trust Senior Research Fellowship (101863). We also acknowledge core support from the Wellcome Trust (092096) and Cancer Research UK (C6946/A14492). We apologize to the many authors whose work could not be represented here due to limitation in space. The authors declare that they have no competing interests. 


\section{Figure 1: Large-scale chromosome organization}

A: Computational model of the 3D structure of a haploid mouse ES genome using data from a single-cell Hi-C experiment. Left: Modelled arrangement of the chromosomes within a single nucleus. Each chromosome is coloured differently. Center: Cross-section of the modelled nucleus, with A compartment in blue and B compartment in red. The B compartment is enriched at the nuclear lamina and in a central ring that surrounds the nucleolus. Right: Different structural organization of chromosome 9 modelled from two different single-cell Hi-C datasets. Figures extracted from [6].

B: Pearson correlation map of chromatin interactions on Chromosome 17 at a resolution of $500 \mathrm{~kb}$. The Eigenvector obtained by Principal Component Analysis (PCA) reveals segregation of the chromosome in two compartments, A (positive values) and B (negative values). Data visualised using Juicebox and obtained from [4].

C: A/B compartments are present in interphase, lost in mitosis and re-established after cell division. A/B compartment re-establishment could potentially rely on retained chromatin domains defined by histone modifications. The Pearson correlation maps of interactions are coloured as in B. Data obtained from [9] and visualised using Juicebox [4].

\section{Figure 2: Topologically Associated Domain (TAD) organization in mammals}

Three theoretical TADs (green, red and blue) are depicted. 4C tracks from [39] are used to illustrate the "insulating" properties of TAD boundaries ( $4 \mathrm{C}$ experiments assess the interactions between one specific locus and the rest of the genome; the assessed locus in each 4C experiment is indicated by an arrowhead). Insulating loops between TAD boundaries are represented by dashed lines while contacts between regulatory elements are represented by 
solid yellow lines.

\section{Figure 3: Model of dynamic loop extrusion}

A loop extrusion factor (LEF) binds to a segment of chromatin between two boundary factors (BF) located on TAD boundaries and initiates loop extrusion (a). While this loop is growing, a new LEF could bind within the loop (b), leading to the extrusion of a secondary nested loop (c). If BFs are present when the loop ends reach a TAD boundary, the loop is temporarily stabilized (d) then disrupts when a LEF or LEF/BF complex dissociates (e). Alternatively, if a BF is not present, the loop could bypass the TAD boundary (f). Loops could potentially also dissociate during any phase of extrusion. Model based on references [44,45]. 
1. Giorgetti L, Heard E: Closing the loop: 3C versus DNA FISH. Genome Biol 2016, 17:215.

2. Dekker J, Rippe K, Dekker M, Kleckner N: Capturing Chromosome Conformation. Science 2002, 295:1306-1312.

3. Lieberman Aiden E, van Berkum NL, Williams L, Imakaev M, Ragoczy T, Telling A, Amit I, Lajoie BR, Sabo PJ, Dorschner MO, et al.: Comprehensive mapping of longrange interactions reveals folding principles of the human genome. Science 2009, 326:289-93.

4. Rao SSP, Huntley MHH, Durand NCC, Stamenova EKK, Bochkov IDD, Robinson JTT, Sanborn AL, Machol I, Omer ADD, Lander ESS, et al.: A 3D Map of the Human Genome at Kilobase Resolution Reveals Principles of Chromatin Looping. Cell 2014, 159:1665-1680.

5. Croft JA, Bridger JM, Boyle S, Perry P, Teague P, Bickmore WA: Differences in the localization and morphology of chromosomes in the human nucleus. $J$ Cell Biol 1999, 145:1119-31.

6. Stevens TJ, Lando D, Basu S, Atkinson LP, Cao Y, Lee SF, Leeb M, Wohlfahrt KJ, Boucher $\mathrm{W}$, O'Shaughnessy-Kirwan A, et al.: 3D structures of individual mammalian genomes studied by single-cell Hi-C. Nature 2017, 544:59-64.

** This work used single-cell Hi-C coupled with imaging to calculate 3D structures of eight individual mESC genomes. The results show that there is substantial cell-to-cell variability in chromosome structure, but $\mathrm{A} / \mathrm{B}$ compartments have a consistent organization in the nucleus, suggesting that they may drive genome folding.

7. Meister P, Towbin BD, Pike BL, Ponti a, Gasser SM: The spatial dynamics of tissue-specific promoters during C-elegans development. Genes Dev 2010, 24:766782.

8. Kind J, Pagie L, Ortabozkoyun H, Boyle S, Vries SS De, Janssen H, Amendola M, Nolen LD, Bickmore WA, Steensel B Van: Single-Cell Dynamics of GenomeNuclear Lamina Interactions. Cell 2013, 153:178-192.

9. Naumova N, Imakaev M, Fudenberg G, Zhan Y, Lajoie BR, Mirny L, Dekker J: Organization of the mitotic chromosome. Science 2013, 342:948-53.

10. Gibcus JH, Samejima K, Goloborodko A, Samejima I, Naumova N, Nuebler J, Kanemaki M, Xie L, Paulson JR, Earnshaw WC, et al.: A pathway for mitotic chromosome formation. Science 2018, 6135.

11. Evans KJ, Huang N, Stempor P, Chesney MA, Down TA, Ahringer J: Stable Caenorhabditis elegans chromatin domains separate broadly expressed and developmentally regulated genes. Proc Natl Acad Sci 2016, 113:E7020-E7029.

12. Prakash K, Fournier D, Redl S, Best G, Borsos M, Tiwari VK, Tachibana-Konwalski $\mathrm{K}$, Ketting RF, Parekh SH, Cremer C, et al.: Superresolution imaging reveals structurally distinct periodic patterns of chromatin along pachytene chromosomes. Proc Natl Acad Sci U S A 2015, 112.

13. Boettiger AN, Bintu B, Moffitt JR, Wang S, Beliveau BJ, Fudenberg G, Imakaev M, Mirny $\mathrm{L}$, Wu C, Zhuang $\mathrm{X}$ : Super-resolution imaging reveals distinct chromatin folding for different epigenetic states. Nature 2016, 529:1-15.

* This work used super-resolution microscopy in D. melanogaster Kc167 cells to investigate 3D structure of chromatin in different epigenetic states, showing that active, inactive, and Polycomb repressed chromatin have distinct packing properties. Polycomb chromatin has the densest packing and excludes active chromatin more strongly than inactive chromatin. 
14. Wijchers PJ, Krijger PHL, Geeven G, Zhu Y, Denker A, Verstegen MJAM, ValdesQuezada C, Vermeulen C, Janssen M, Teunissen H, et al.: Cause and Consequence of Tethering a SubTAD to Different Nuclear Compartments. Mol Cell 2016, 61:461473.

15. Gaydos LJ, Wang W, Strome S: H3K27me and PRC2 transmit a memory of repression across generations and during development. Science 2014, 345:15151518.

16. Rao SSP, Huang S-C, Hilaire BGS, Engreitz JM, Perez EM, Kieffer-Kwon K-R, Sanborn AL, Johnstone SE, Bascom GD, Bochkov ID, et al.: Cohesin Loss

Eliminates All Loop Domains. Cell 2017, 171:305-320.e24.

** This paper, together with $[54,56]$, remove CTCF, cohesin, or the cohesin release factor WAPL in mammalian cells showed that CTCF and cohesin are essential for TAD formation, but not compartment structure.

17. Carelli FN, Sharma G, Ahringer J: Broad Chromatin Domains : An Important Facet of Genome Regulation. BioEssays 2017, 1700124:1-7.

18. Rowley MJ, Nichols MH, Lyu X, Ando-Kuri M, Rivera ISM, Hermetz K, Wang P, Ruan Y, Corces VG: Evolutionarily Conserved Principles Predict 3D Chromatin Organization. Mol Cell 2017, 67:837-852.e7.

** This study uses high resolution chromatin interaction methods to show that the $D$. melanogaster genome is locally organized into "compartmental domains" that correspond with A/B compartments. Through analyses of the genomes of other organisms, the authors suggest that compartmental domains are play a major role in genome organization in eukaryotes.

19. Maeshima K, Ide S, Hibino K, Sasai M: Liquid-like behavior of chromatin. Curr Opin Genet Dev 2016, 37:36-45.

20. Feric M, Vaidya N, Harmon TS, Kriwacki RW, Pappu R V, Brangwynne CP, Mitrea DM, Zhu L, Richardson TM: Coexisting Liquid Phases Underlie Nucleolar Subcompartments. Cell 2016, 165:1686-1697.

21. Larson AG, Elnatan D, Keenen MM, Trnka MJ, Johnston JB, Burlingame AL, Agard DA, Redding S, Narlikar GJ: Liquid droplet formation by HP1 $\alpha$ suggests a role for phase separation in heterochromatin. Nat Publ Gr 2017, 547.

22. Strom AR, Emelyanov A V, Mir M, Fyodorov D V, Darzacq X, Karpen GH: Phase separation drives heterochromatin domain formation. Nat Publ Gr 2017, doi:10.1038/nature22989.

23. Falahati H, Pelham-Webb B, Blythe S, Wieschaus EF: Nucleation by rRNA Dictates the Precision of Nucleolus Assembly. Curr Biol 2016, doi:10.1016/j.cub.2015.11.065.

24. Dixon JR, Selvaraj S, Yue F, Kim A, Li Y, Shen Y, Hu M, Liu JS, Ren B: Topological domains in mammalian genomes identified by analysis of chromatin interactions. Nature 2012, 485:376-380.

25. Nora EP, Lajoie BR, Schulz EG, Giorgetti L, Okamoto I, Servant N, Piolot T, van Berkum NL, Meisig J, Sedat J, et al.: Spatial partitioning of the regulatory landscape of the X-inactivation centre. Nature 2012, 485:381-385.

26. Sexton T, Yaffe E, Kenigsberg E, Bantignies F, Leblanc B, Hoichman M, Parrinello $\mathrm{H}$, Tanay A, Cavalli G: Three-dimensional folding and functional organization principles of the Drosophila genome. Cell 2012, 148:458-472.

27. Phillips-Cremins JE, Sauria MEG, Sanyal A, Gerasimova TI, Lajoie BR, Bell JSK, Ong C-T, Hookway TA, Guo C, Sun Y, et al.: Architectural Protein Subclasses Shape 3D Organization of Genomes during Lineage Commitment. Cell 2013, 
doi:10.1016/j.cell.2013.04.053.

28. Dowen JM, Fan ZP, Hnisz D, Ren G, Abraham BJ, Zhang LN, Weintraub AS, Schuijers J, Lee TI, Zhao K, et al.: Control of Cell Identity Genes Occurs in Insulated Neighborhoods in Mammalian Chromosomes. Cell 2014, doi:10.1016/j.cell.2014.09.030.

29. Pope BD, Ryba T, Dileep V, Yue F, Wu W, Denas O, Vera DL, Wang Y, Hansen RS, Canfield TK, et al.: Topologically associating domains are stable units of replication-timing regulation. Nature 2014, 515:402-405.

30. Symmons O, Uslu VV, Tsujimura T, Ruf S, Nassari S, Schwarzer W, Ettwiller L, Xois Spitz F, Spitz F: Functional and topological characteristics of mammalian regulatory domains. Genome Res 2014, 24:390-400.

31. Phanstiel DH, Van Bortle K, Spacek DV, Hess GT, Saad Shamim M, Machol I, Love MI, Lieberman Aiden E, Bassik MC, Snyder MP: Static And Dynamic DNA Loops Form AP-1 Bound Activation Hubs During Macrophage Development. Mol Cell 2017, doi:10.1101/142026.

32. Rubin AJ, Barajas BC, Furlan-Magaril M, Lopez-Pajares V, Mumbach MR, Howard I, Kim DS, Boxer LD, Cairns J, Spivakov M, et al.: Lineage-specific dynamic and preestablished enhancer-promoter contacts cooperate in terminal differentiation. Nat Genet 2017, doi:10.1038/ng.3935.

33. Beagan JA, Duong MT, Titus KR, Zhou L, Cao Z, Ma J, Lachanski C V., Gillis DR, Phillips-Cremins JE: YY1 and CTCF orchestrate a 3D chromatin looping switch during early neural lineage commitment. Genome Res 2017, 27:1139-1152.

34. Rudan MV, Barrington C, Henderson S, Ernst C, Odom DT, Tanay A, Hadjur S: Comparative Hi-C Reveals that CTCF Underlies Evolution of Chromosomal Domain Architecture. Cell Rep 2015, 10:1297-1309.

35. Bell O, Tiwari VK, Thomä NH, Schübeler D: Determinants and dynamics of genome accessibility. Nat Rev Genet 2011, 12:554-64.

36. Guo Y, Xu Q, Canzio D, Krainer AR, Maniatis T, Wu Q: CRISPR Inversion of CTCF Sites Alters Genome Topology and Enhancer/Promoter Function. Cell 2015, 162:900-910.

37. de Wit E, Vos ESM, Holwerda SJB, Valdes-Quezada C, Verstegen MJAM, Teunissen H, Splinter E, Wijchers PJ, Krijger PHL, de Laat W: CTCF Binding Polarity Determines Chromatin Looping. Mol Cell 2015, 60:676-684.

38. Narendra V, Bulajic M, Dekker J, Mazzoni EO, Reinberg D: CTCF-mediated topological boundaries during development foster appropriate gene regulation. Genes Dev 2016, 30:2657-2662.

39. Lupiáñez DG, Kraft K, Heinrich V, Krawitz P, Brancati F, Klopocki E, Horn D, Kayserili H, Opitz JM, Laxova R, et al.: Disruptions of topological chromatin domains cause pathogenic rewiring of gene-enhancer interactions. Cell 2015, 161:1012-1025.

40. Lee DP, Lek Wen Tan W, George Anene-Nzelu C, Yiqing Li P, Anh Luu Danh T, Tiang Z, Ling Ng S, Autio MI, Jiang J, Fullwood M, et al.: Gene neighbourhood integrity disrupted by CTCF loss in vivo. Biorxiv 2017, doi:10.1101/187393.

41. Franke M, Ibrahim DM, Andrey G, Schwarzer W, Heinrich V, Schöpflin R, Kraft K, Kempfer R, Jerković I, Chan W-L, et al.: Formation of new chromatin domains determines pathogenicity of genomic duplications. Nature 2016, 538:265-269.

* This work investigating spatial organization of the Sox9 neighbourhood in mice and humans demonstrates that genomic duplications introducing new TAD boundaries can have pathological effects.

42. Hnisz D, Weintraub AS, Day DS, Valton A-L, Bak RO, Li CH, Goldmann J, Lajoie 
BR, Fan ZP, Sigova AA, et al.: Activation of proto-oncogenes by disruption of chromosome neighborhoods. Science 2016, 351:1454-1458.

43. Merkenschlager M, Nora EP: CTCF and Cohesin in Genome Folding and Transcriptional Gene Regulation. Annu Rev Genomics Hum Genet 2016, 17:17-43.

44. Sanborn AL, Rao SSP, Huang S-C, Durand NC, Huntley MH, Jewett AI, Bochkov ID, Chinnappan D, Cutkosky A, Li J, et al.: Chromatin extrusion explains key features of loop and domain formation in wild-type and engineered genomes. Proc Natl Acad Sci 2015, 112:201518552.

45. Fudenberg G, Imakaev M, Lu C, Goloborodko A, Abdennur N, Mirny L: Formation of Chromosomal Domains by Loop Extrusion. Cell Rep 2016, 15:2038-49.

* These papers $[44,45]$ propose a loop extrusion model based on computational modelling to explain the mechanism of formation of TADs. The models explain many biological observations (e.g. loop peaks at TAD boundaries, nested TADs, CTCF motif orientation) and highlights the dynamics of loop formation.

46. Uusküla-reimand L, Hou H, Samavarchi-tehrani P, Rudan MV, Liang M, Medinarivera A, Mohammed $\mathrm{H}$, Schmidt $\mathrm{D}$, Schwalie P, Young EJ, et al.: Topoisomerase II beta interacts with cohesin and CTCF at topological domain borders. Genome Biol 2016, doi:10.1186/s 13059-016-1043-8.

47. Barrington C, Finn R, Hadjur S: Cohesin biology meets the loop extrusion model. Chromosom Res 2017, doi:10.1007/s10577-017-9550-3.

48. Racko D, Benedetti F, Dorier J, Stasiak A: Transcription-induced supercoiling as the driving force of chromatin loop extrusion during formation of TADs in interphase chromosomes. Nucleic Acids Res 2017,

49. Hug CB, Grimaldi AG, Kruse K, Vaquerizas JM: Chromatin Architecture Emerges during Zygotic Genome Activation Independent of Transcription. Cell 2017, 169:216-228.e19.

50. Ke Y, Xu Y, Chen X, Feng S, Liu Z, Sun Y, Yao X: 3D Chromatin Structures of Mature Gametes and Structural Reprogramming during Mammalian Embryogenesis. Cell 2017, 170:367-381.e20.

51. Hansen AS, Pustova I, Cattoglio C, Tjian R, Darzacq X: CTCF and cohesin regulate chromatin loop stability with distinct dynamics. Elife 2017, 6:1-33.

** Using single-molecule imaging to measure chromatin binding dynamics in mESCs, this paper shows that CTCF and cohesin do not form a stable complex, but instead rapidly exchange on chromatin, with CTCF binding having a much shorter residence time than cohesin. The binding dynamics suggest that chromatin loops are continually forming and breaking.

52. Nagano T, Lubling Y, Stevens TJ, Schoenfelder S, Yaffe E, Dean W, Laue ED, Tanay A, Fraser P: Single-cell Hi-C reveals cell-to-cell variability in chromosome structure. Nature 2013, 502.

53. Flyamer IM, Gassler J, Imakaev M, Brandão HB, Ulianov S V., Abdennur N, Razin S V., Mirny L, Tachibana-Konwalski K: Single-nucleus Hi-C reveals unique chromatin reorganization at oocyte-to-zygote transition. Nature 2017, 544:110 114.

* Single nucleus Hi-C in mouse oocytes and early zygotes shows that the positions of TADs vary substantially between individual cells, supporting their dynamic nature. The authors also observe TADs and loops, but not compartments, in maternal chromatin, suggesting the different structures are formed by different mechanisms. 
54. Nora EP, Goloborodko A, Valton A-L, Dekker J, Mirny L, Gibcus JH, Uebersohn A, Abdennur N, Bruneau BG: Targeted Degradation of CTCF Decouples Local Insulation of Chromosome Domains from Genomic Compartmentalization. Cell 2017, 169:930-944.

** This paper, together with $[16,56]$, remove CTCF, cohesin, or the cohesin release factor WAPL in mammalian cells showed that CTCF and cohesin are essential for TAD formation, but not compartment structure.

55. Schwarzer W, Abdennur N, Goloborodko A, Pekowska A, Fudenberg G, Loe-Mie Y, Fonseca NA, Huber W, Haering C, Mirny L, et al.: Two independent modes of chromatin organization revealed by cohesin removal. Nature 2017, doi:10.1038/nature24281.

56. Haarhuis JHI, van der Weide RH, Blomen VA, Yáñez-Cuna JO, Amendola M, van Ruiten MS, Krijger PHL, Teunissen H, Medema RH, van Steensel B, et al.: The Cohesin Release Factor WAPL Restricts Chromatin Loop Extension. Cell 2017, 169:693-707.e14.

** This paper, together with $[16,54]$, remove CTCF, cohesin, or the cohesin release factor WAPL in mammalian cells showed that CTCF and cohesin are essential for TAD formation, but not compartment structure.

57. Stadler M, Haines JE, Eisen MB: Convergence of topological domain boundaries, insulators, and polytene interbands revealed by high-resolution mapping of chromatin contacts in the early Drosophila melanogaster embryo. Elife 2017, doi:10.1101/149344.

58. Bortle K Van, Nichols MH, Li L, Ong C, Takenaka N, Qin ZS, Corces VG: Insulator function and topological domain border strength scale with architectural protein occupancy. Genoome Biol 2014, doi:10.1186/gb-2014-15-5-r82.

59. Barutcu AR, Lajoie BR, Fritz AJ, Mccord RP, Nickerson JA, Wijnen AJ Van, Lian JB, Stein JL, Dekker J, Stein GS, et al.: SMARCA4 regulates gene expression and higher- order chromatin structure in proliferating mammary epithelial cells. Genome Res 2016, doi:10.1101/gr.201624.115.

60. Dixon JR, Gorkin DU, Ren B: Chromatin Domains: The Unit of Chromosome Organization. Mol Cell 2016, 62:668-680.

61. El-sharnouby S, Fischer B, Magbanua JP, Umans B, Flower R, Choo SW, Russell S, White R: Regions of very low H3K27me3 partition the Drosophila genome into topological domains. PLoS One 2017, doi:10.1371/journal.pone.0172725.

* The study shows a correspondence between genome organization and domains of high and low H3K27me3 levels in Drosophila sperm. High H3K27me3 levels correspond with TADs and low levels with boundaries that are enriched for housekeeping genes.

62. Crane E, Bian Q, McCord RP, Lajoie BR, Wheeler BS, Ralston EJ, Uzawa S, Dekker $\mathrm{J}$, Meyer BJ: Condensin-driven remodelling of $\mathbf{X}$ chromosome topology during dosage compensation. Nature 2015, 523:240-244.

63. Liu C, Wang C, Wang G, Becker C, Zaidem M, Weigel D: Genome-wide analysis of chromatin packing in Arabidopsis thaliana at single-gene resolution. Genome Res 2016, doi:10.1101/gr.204032.116.

64. Ghavi-Helm Y, Klein FA, Pakozdi T, Ciglar L, Noordermeer D, Huber W, Furlong EEM: Enhancer loops appear stable during development and are associated with paused polymerase. Nature 2014, 512:96-100.

65. Mifsud B, Tavares-Cadete F, Young AN, Sugar R, Schoenfelder S, Ferreira L, Wingett 
SW, Andrews S, Grey W, Ewels PA, et al.: Mapping long-range promoter contacts in human cells with high-resolution capture Hi-C. Nat Genet 2015, 47:598-606.

66. Li G, Ruan X, Auerbach RK, Sandhu KS, Zheng M, Wang P, Poh HM, Goh Y, Lim J, Zhang J, et al.: Extensive promoter-centered chromatin interactions provide a topological basis for transcription regulation. Cell 2012, 148:84-98.

67. Hsieh T-HS, Fudenberg G, Goloborodko A, Rando OJ: Micro-c XL: assaying chromosome conformation from the nucleosome to the entire genome. Nat Methods 2016, doi:10.1038/nMeth.4025.

68. Schmitt AD, Hu M, Jung I, Lin Y, Barr CL, Ren B: A Compendium of Chromatin Contact Maps Reveals Spatially Active Regions in the Human Genome. Cell Rep 2016, doi:10.1016/j.celrep.2016.10.061.

69. Schoenfelder S, Furlan-magaril M, Mifsud B, Tavares-cadete F, Sugar R, Javierre BM, Nagano T, Katsman Y, Sakthidevi M, Wingett SW, et al.: The pluripotent regulatory circuitry connecting promoters to their long-range interacting elements. Genome Res 2015, doi:10.1101/gr.185272.114.

70. Deng W, Lee J, Wang H, Miller J, Reik A, Gregory PD, Dean A, Blobel GA: Controlling Long-Range Genomic Interactions at a Native Locus by Targeted Tethering of a Looping Factor. Cell 2012, 149:1233-1244.

71. Deng W, Rupon JW, Krivega I, Breda L, Motta I, Jahn KS, Reik A, Gregory PD, Rivella S, Dean A, et al.: Reactivation of Developmentally Silenced Globin Genes by Forced Chromatin Looping. Cell 2014, 158:849-860. 

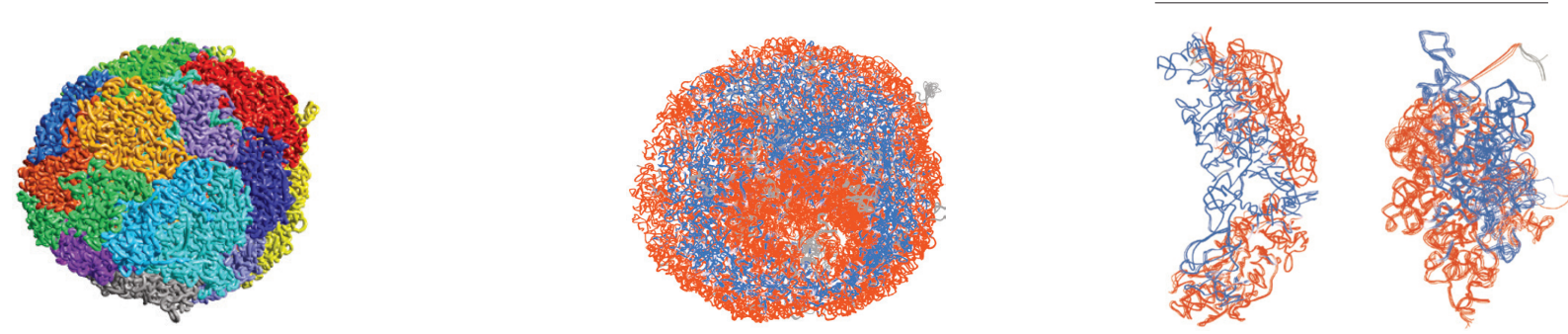

A / B compartments

B

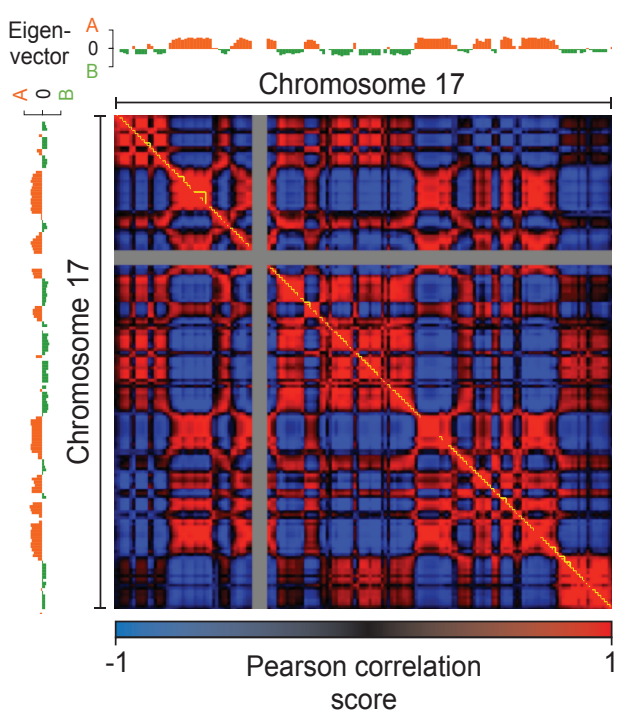

C

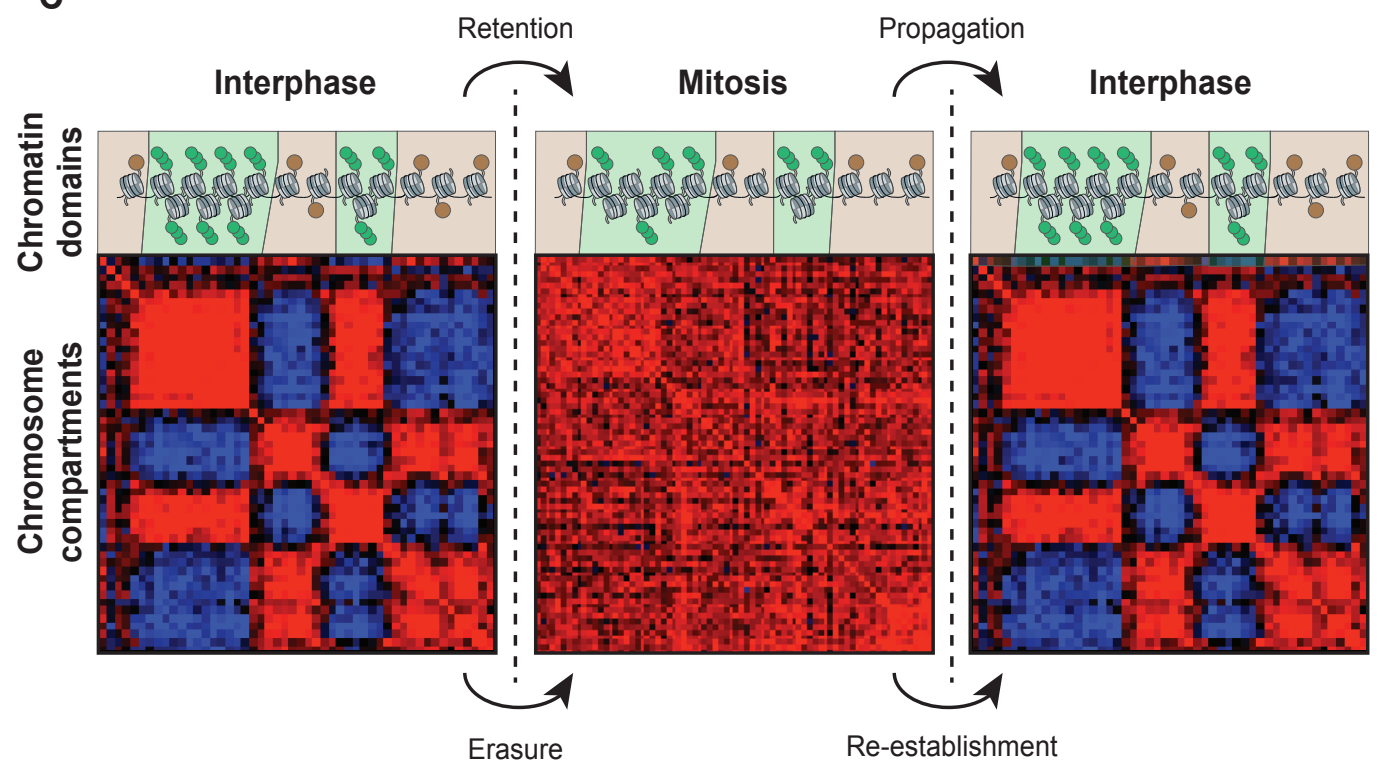




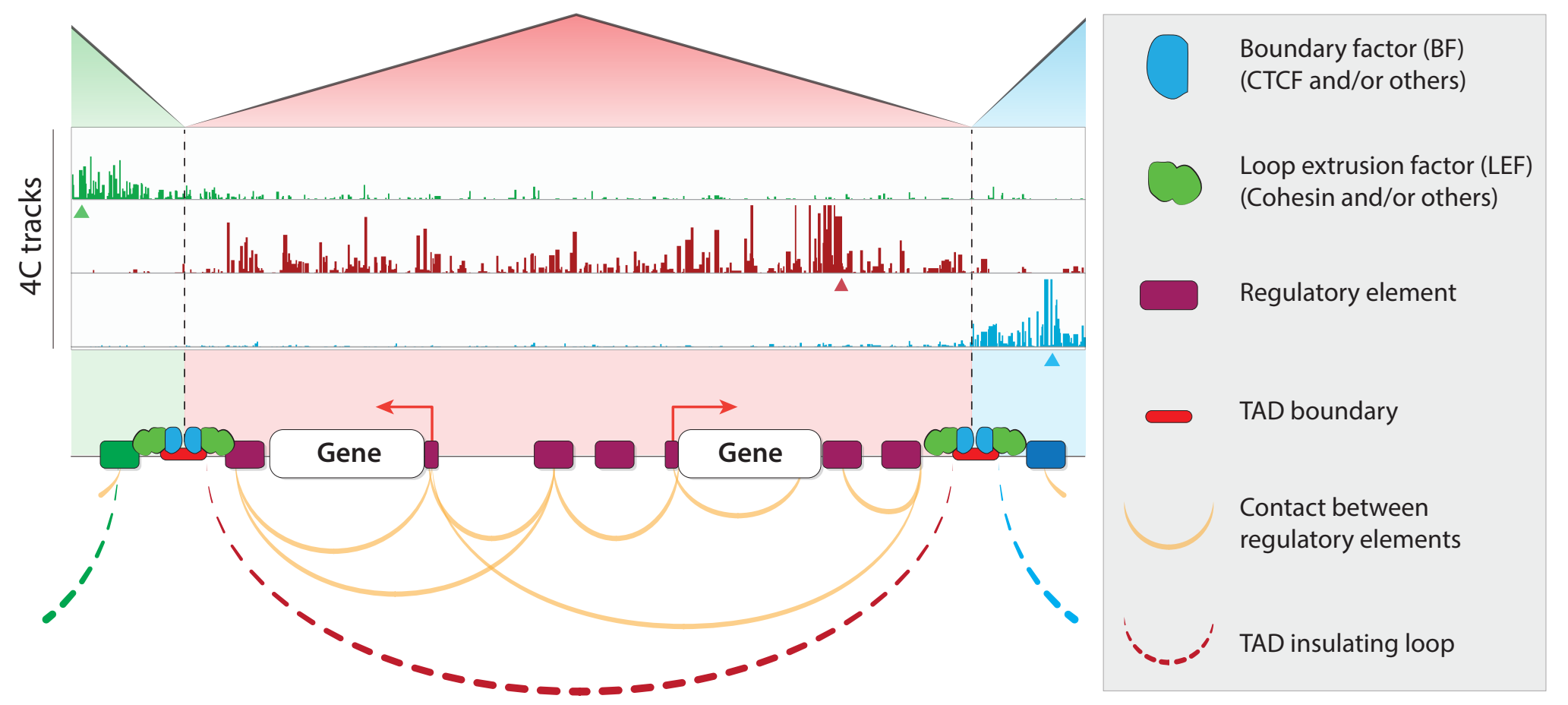




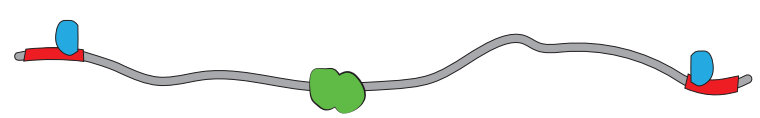

(a) Initiation of loop

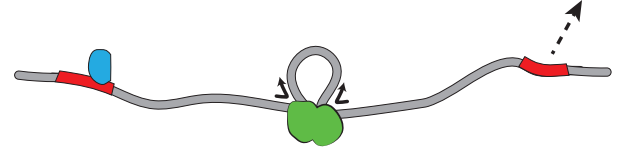

(b) Binding of a new LEF

(c) Initiation of nested loop
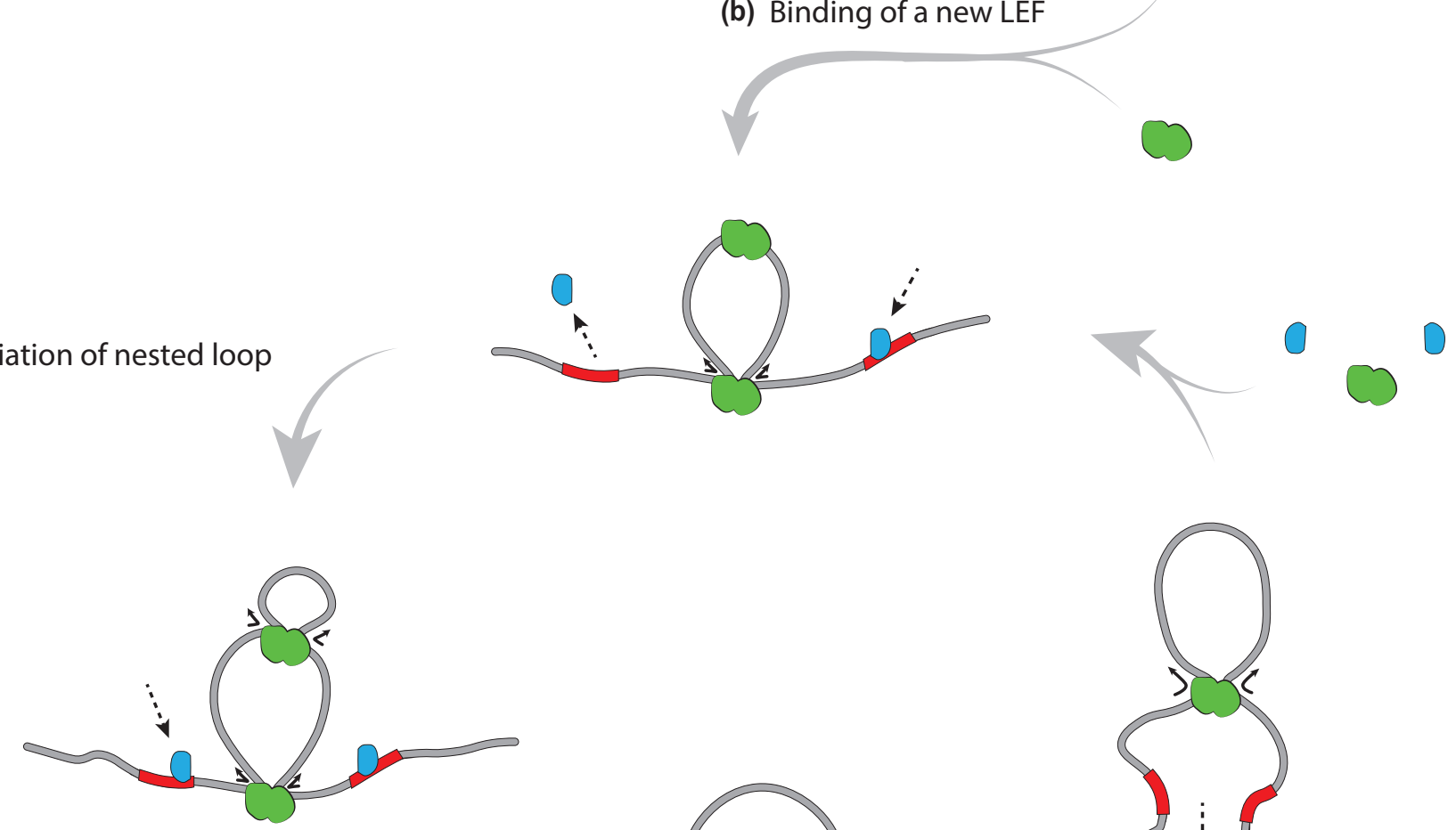

(d) Transient loop stabilization

(f) BF dissociation
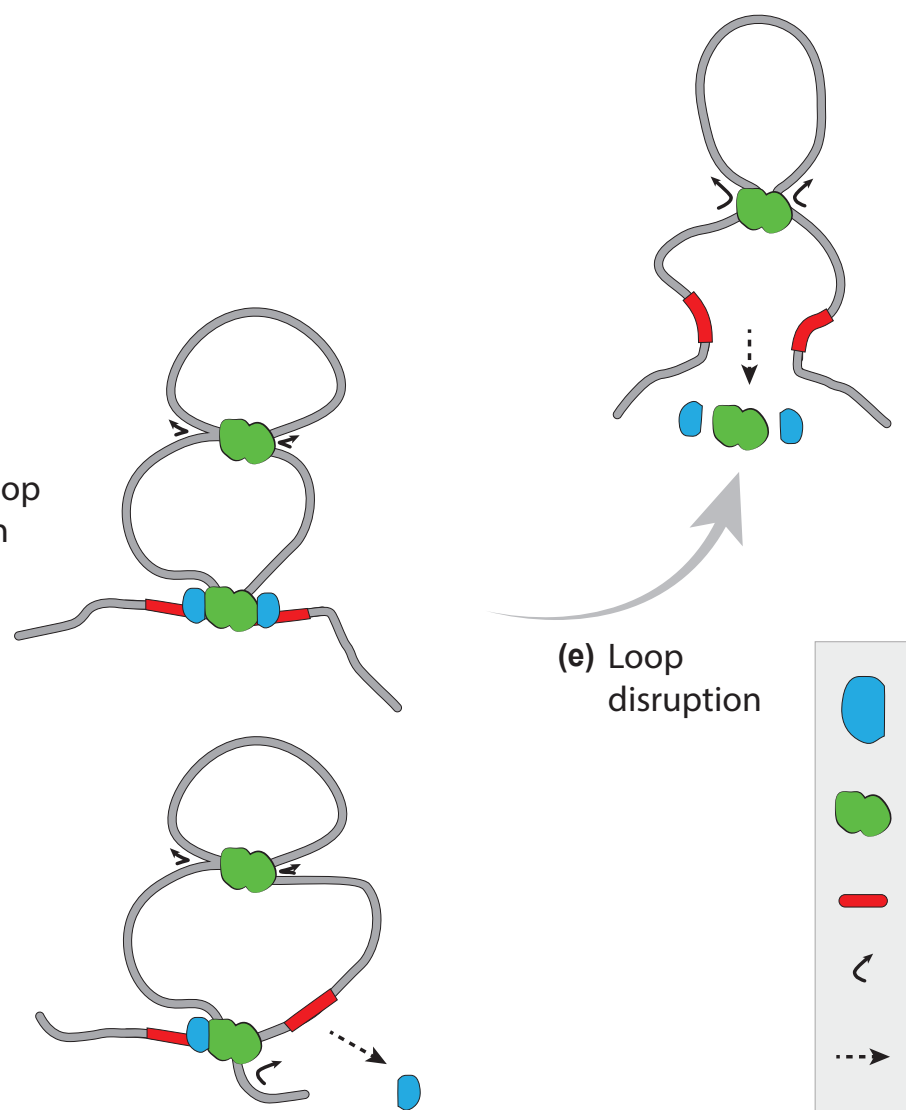

\section{(e) Loop disruption}

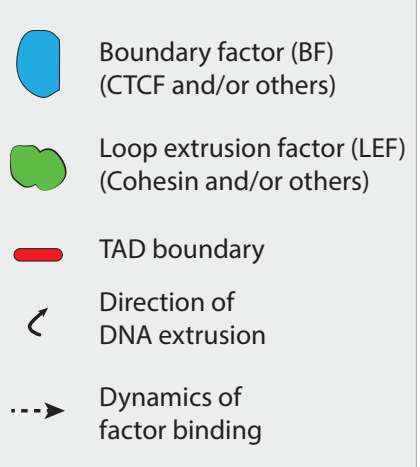

